\title{
LAS REDES DE APOYO SOCIAL ONLINE Y OFFLINE EN LOS INMIGRANTES DE MÁLAGA (ESPAÑA)*
}

\section{THE ONLINE AND OFFLINE SOCIAL SUPPORT NETWORKS FOR IMMIGRANTS IN MÁLAGA (SPAIN)}

Mario Millán-Franco**, LUis Gómez-Jacinto***, María Isabel Hombrados-Mendieta ${ }^{\star \star \star *}$, Alba García-Cid ${ }^{\star \star \star \star *}$

Resumen: Como consecuencia del desarrollo de las Tecnologías de la Información y la Comunicación el aumento de la velocidad de conexión de los inmigrantes internacionales con sus países de origen y destino ha supuesto un hito de notables dimensiones. Esta investigación analiza cómo las redes de apoyo online y offline influyen en la percepción de apoyo social, sentido de comunidad y satisfacción vital de los inmigrantes. La metodología utilizada es de corte cuantitativo, con una muestra representativa de 1563 inmigrantes residentes en Málaga (España). Los resultados evidencian el beneficio que supone para los inmigrantes disponer de una

* Agradecimientos:

Este estudio fue posible gracias a las subvenciones del Ministerio de Economía y Competitividad de España (Proyectos I+D: PSI2013-40508-P y PSI2017-85941-R). Mario Millán Franco señala que este artículo se ha elaborado durante las estancias de investigación realizadas en la Pontificia Universidad Católica del Perú (Lima) y en la Università degli Studi di Palermo (Italia), financiadas respectivamente por el Plan Propio de Investigación y Transferencia de la Universidad de Málaga y por el Ministerio de Ciencia, Innovación y Universidades de España.

** Profesor de Trabajo Social y Servicios Sociales de la Universidad de Málaga,mmillan@uma.es

*** Profesor de Psicología Social de la Universidad de Málaga

**** Profesora de Psicología Social de la Universidad de Málaga

***** Investigadora en Formación de la Universidad de Málaga 
red de apoyo amplia y heterogénea, posicionándose como un elemento de gran valor para la percepción de apoyo social, sentido de comunidad y satisfacción vital. Los hallazgos defienden la funcionalidad de la comunicación mediada por la tecnología durante la aculturación y la necesidad de ayudar a los inmigrantes a construir sus propias redes sociales.

Palabras clave: comunicación mediada por la tecnología (CMT); inmigración; apoyo social; sentido de comunidad; redes sociales.

Abstract: Thanks to the development of Information and Communication Technologies, international immigrants now have available fast connectivity with their countries of origin and destination. This study analyzes how online and offline support networks influence the perception of social support, sense of community, and life satisfaction of immigrants. A quantitative approach was applied using a representative sample of 1563 immigrants residing in Malaga (Spain). The results show that immigrants benefit from having a wide and heterogeneous support network, and that the Internet is of great value in relation to the perception of social support, sense of community, and life satisfaction. The findings support the usefulness of technology-mediated communication during the acculturation process and the need to help immigrants build their own social networks.

Key words: technologically mediated communication; immigration; social support; sense of community; social networks.

\section{INTRODUCCIÓN}

En el siglo XXI como consecuencia del desarrollo de las Tecnologías de la Información y la Comunicación (TICs) el aumento de la velocidad de conexión de los inmigrantes internacionales con sus países de origen y destino ha supuesto un hito de notables dimensiones (Peng, 2016; Viruela, 2007). Los inmigrantes usan las TICs tanto como las poblaciones locales, lo que explicita su grado de motivación y concienciación hacia las potencialidades del medio digital (Codagnone y Kluzer, 2011). Internet y el avance de la comunicación mediada por la tecnología (CMT) está permitiendo cada vez más a los inmigrantes brindar o recibir apoyo social de sus familias o amigos (Chen y Choi, 2011). Pocos estudios se han centrado en analizar cuáles son los 
efectos de las redes sociales de apoyo virtuales en la calidad de vida de los inmigrantes. Aunque el uso de las TICs e Internet en términos generales conlleva consecuencias positivas en la vida de las personas, a día de hoy, existen opiniones enfrentadas en torno a sus beneficios. Esta investigación se focaliza en un uso concreto de Internet, la comunicación por Internet, referido a la frecuencia e intensidad con la que se usa Internet para establecer y mantener comunicación entre personas. El desarrollo de las nuevas tecnologías demanda profundizar en el análisis de la influencia del uso de Internet en el bienestar de la población (Valkenburg y Peter, 2007). Así, es necesario conocer las implicaciones de la red social de apoyo de los inmigrantes en su percepción de apoyo social, sentido de comunidad y satisfacción vital (Chen y Choi, 2011). Las TICs han revolucionado las formas en las que las personas se comunican y reciben apoyo social beneficioso para su bienestar. Esto en la actualidad ha provocado una notoria redefinición del concepto "comunidad", desarrollándose paulatinamente una evolución desde una situación donde la proximidad territorial de sus miembros era de una importancia capital hacia una perspectiva donde destaca la relevancia de la conexión a través de las TICs.

El apoyo social, la satisfacción vital y el sentido de comunidad se han relacionado con las redes sociales offline; mientras que son incipientes los estudios que tratan simultáneamente la influencia de las redes de apoyo social, online y offline, en dichas variables psicosociales. Esta investigación incluye en el contexto de los inmigrantes de Málaga el análisis de la influencia de las nuevas tecnologías para mantenerse conectados en la sociedad de origen y destino, a la par que analiza las relaciones interculturales que vivencian en el contexto de acogida. Se estudia la potencial influencia de la composición de las redes de apoyo, online y offline, en la percepción de apoyo social, sentido de comunidad y satisfacción vital de los inmigrantes.

\section{MARCO TEÓRICO}

\section{La migración y la comunicación mediada por la tecnología}

El desarrollo de las TICs ha provocado que la vivencia del mundo virtual sea algo cotidiano y paralelo a la vida del ser humano. El uso 
de Internet que destaca por su capacidad para modular la interacción social y, por tanto, el bienestar de las personas se denomina Internet communication (Valkenburg y Peter, 2007). De hecho, hay más probabilidad de que se desarrolle esta influencia sobre el bienestar y el apoyo social percibido durante procesos como la migración, caracterizados por una alta vulnerabilidad. Los migrantes internacionales necesitan apoyo social para enfrentarse a un entorno social desconocido, normalmente separados de sus familias (Chib, Wilkin y Hua, 2013). Cada día es más frecuente que los inmigrantes usen las redes virtuales para mantenerse conectados con su país de origen y destino (Diminescu, 2008). Se ha demostrado el éxito de la CMT para conservar el contacto con los amigos y la familia, posibilitando incluso una cierta presencia en las comunidades de origen al suponer la ruptura del límite espacio-temporal humano (Francisco, 2015). En un contexto nuevo las TICs proporcionan a los inmigrantes un recurso importante para consolidar vínculos con las personas que forman parte del área donde desarrollan sus vidas (Castro y González, 2008).

\section{Modelos teóricos sobre la influencia de la comunicación mediada por la tecnología en las relaciones interpersonales}

El análisis de los beneficios e inconvenientes, y los mecanismos de actuación, del progresivo uso de las TICs e Internet en la comunicación interpersonal, incluyendo el ámbito migratorio, ha sido un tema de amplio debate en los últimos años. Así, las propuestas de modelos teóricos que tratan de explicar la influencia de la CMT en las relaciones interpersonales han sido diversas. Nie, Hillygus y Erbring (2002) señalan tres modelos teóricos que explican el efecto del uso de Internet en la cantidad y calidad de la comunicación interpersonal y la sociabilidad. En primer lugar, la hipótesis de la eficiencia sostiene que Internet posibilita nuevas opciones para mantener, complementar y crear redes sociales. La CMT complementaría en lugar de reemplazar el apoyo offline de los inmigrantes (Chen y Choi, 2011). La hipótesis de desplazamiento defiende que el tiempo destinado a una actividad no se puede gastar en otras. El tiempo en línea es una actividad social que compite con el tiempo social cara a cara, no entendiéndose como complementarios. Por último, la hipótesis comunitaria señala 
que los efectos del uso de Internet están más influenciados por la calidad del tiempo destinado que por la cantidad del mismo. Esta perspectiva focaliza su atención en la capacidad de Internet para eliminar la distancia y generar y mantener vínculos sobre la base de un interés compartido.

Recientemente la teoría de Polymedia ha planteado en el ámbito migratorio que lo verdaderamente importante en torno a la comunicación interpersonal son las elecciones que las personas hacen entre los diferentes medios digitales a los que tienen acceso para comunicarse; entendiendo que el uso de los diversos medios digitales está influenciado por el vínculo entre los comunicantes (Madianou y Miller, 2013).

La hipótesis del contacto se ha ampliado en los últimos años para incluir la CMT generando resultados contradictorios, si bien se ha señalado que con las condiciones idóneas el contacto intergrupal a través de cualquier medio potencialmente puede reducir los prejuicios (Walther, Hoter, Ganayem y Shonfeld, 2015). En última instancia, el concepto de capital social se ha presentado en los últimos tiempos como un elemento útil para analizar los efectos del uso de las TICs por parte de los inmigrantes en las relaciones interpersonales, por lo que podría entenderse como un modelo en sí mismo.

\section{Las redes sociales cómo medio de trasmisión de apoyo social}

Fruto del desarrollo de las herramientas para establecer, mantener y consolidar la comunicación humana en los últimos años el contexto donde se desarrollan las relaciones sociales ha variado ostensiblemente, modificando a su vez la naturaleza de las interacciones interpersonales y la propia red de relaciones (Dunbar, 2016; González, Zayas y Guil, 2015). Desde inicios del siglo XXI el estudio de la construcción de la red social, incluyendo las implicaciones de las redes sociales online, y más recientemente sus repercusiones en la adaptación y bienestar de las personas se ha convertido en una cuestión de vital importancia (Llopis, 2005). La migración conlleva un debilitamiento de las redes sociales, actuando generalmente como un potente estresor que tiene consecuencias importantes en la calidad de vida y en la inclusión social de los inmigrantes (Garcini et al., 2016; Guruge, Thomson, George y Chaze, 2015). La red 
social de la persona inmigrante en sentido amplio es el medio por el que se trasmite y recibe apoyo social, con independencia de si la interacción es presencial o virtual, por lo que estos son dos conceptos fuertemente relacionados. Así, el propio apoyo social se refiere a "la percepción o la experiencia de que uno es amado y cuidado, estimado y valorado, y es parte de una red social de asistencia mutua y obligaciones" (Taylor et al., 2004, pp. 354-355). La estructura de la red social de las personas inmigrantes se constituye como un elemento fundamental para su bienestar e integración social al situarse como un recurso de primer orden en multitud de situaciones tales como el acceso al empleo, vivienda o apoyo emocional (Ferrer, Palacio, Hoyos y Madariaga, 2014).

La teoría de apoyo social destaca que la interacción de procesos de comunicación dentro de una red social promueve el bienestar psicológico de los individuos (Chib et al., 2013). El apoyo social se encuentra positivamente relacionado con los vínculos de interacción social en contextos tanto offline como online (Wang y Wang, 2013). La CMT posibilita el enriquecimiento de las relaciones sociales y permite el desarrollo y la consolidación de la red social de los inmigrantes (Francisco, 2015).

La existencia de apoyo social depende en primer lugar del tamaño y la calidad de las redes sociales, que provee oportunidades al individuo para interactuar con otros (Martínez de Ibarreta, Redondo y Rúa, 2011; Wong, Ng y Chou, 2017). Así, contar con personas de confianza para expresar emociones, problemas o escuchar su opinión tiene un impacto positivo a la hora de afrontar situaciones difíciles (Lin y Ensel, 1989). Esto es clave en la población inmigrante dado que las redes sociales en sentido amplio les proporcionan recursos adaptados a sus necesidades, a través de la interacción tanto presencial como virtual, y facilitan la adaptación y la mejora de la calidad de vida del colectivo. La importancia del tamaño de la red virtual en el apoyo social online percibido se ha constatado en buscadores de apoyo (Eastin y LaRose, 2005). Un gran tamaño de red online y una alta heterogeneidad de las conexiones demuestran ser favorecedores del apoyo informativo (Trepte, Dienlin y Reinecke, 2015). Se ha señalado una relación positiva entre el número de contactos de Facebook de estudiantes universitarios y la satisfacción vital (Lee, Lee y Kwon, 2011). La satisfacción vital es un constructo muy relacionado con la inclusión social de las personas inmigrantes. Este es considerado como un juicio global que la perso- 
na realiza sobre sus circunstancias existenciales por medio de una comparación entre sus logros obtenidos y sus expectativas de buena vida (Angner, 2010). Se ha apuntado la importancia de la estructura de la red social de los individuos en la determinación del sentido de comunidad hacia el lugar donde desarrollan sus vidas (Maya, 2004). El sentido de comunidad se define como la "percepción de similitud con los demás, una interdependencia consciente con los otros, una voluntad de mantener esta interdependencia dando o haciendo a otros lo que se espera de ellos [y] el sentimiento de que se es parte de una estructura mayor y estable de la que se depende" (Sarason, 1974, p. 157)". El desarrollo del sentido de comunidad está profundamente interconectado con la implicación del sujeto en su entorno meso-social inmediato, con la participación, con la comunicación y con el fortalecimiento comunitario (Ramos-Vidal y Maya-Jariego, 2014). La comunidad se puede entender como "una red de relaciones de apoyo mutuo de la que uno puede depender" (Sarason, 1974, p. 1), en donde el entorno en el que las personas inmigrantes desarrollan sus vidas y el uso de las TICs e Internet posibilitan la construcción de sus redes sociales y el sentido de comunidad (Comeforo, 2016).

El estudio de las redes sociales de apoyo entre los inmigrantes en España es un campo en expansión, con aportaciones desde distintos enfoques y más recientemente de carácter cuantitativo (Martínez de Ibarreta et al., 2011). Entre ellas, las investigaciones de De Miguel Luken y Tranmer (2010), enfocada en los modelos multinivel, y de Álvarez de Sotomayor (2008), sobre la influencia de las redes étnicas en el éxito educativo de hijos de inmigrantes. La naturaleza protectora de las redes de apoyo social en inmigrantes se ha demostrado en diversos ámbitos como la incidencia y evolución de enfermedades mentales (Salinero-Fort, Jiménez-García, De Burgos-Lunar, Chico-Moraleja y Gómez-Campelo, 2015).

\section{Dimensiones del apoyo social}

El apoyo social es un constructo complejo y multidimensional en el que se incluyen tres dimensiones: estructural, funcional y calidad percibida (Lynch, 1998). El apoyo social puede definirse como el conjunto de provisiones expresivas o instrumentales - percibidas o recibidas- proporcionadas por la comunidad, las redes sociales 
y las personas de confianza, las cuales se pueden producir tanto en situaciones cotidianas como en momentos de crisis (Lin, 1986). La dimensión estructural se refiere a las características objetivas de la red de apoyo social, v.g. el tamaño o el número de miembros, obteniéndose información cuantitativa sobre la potencial disponibilidad de apoyo. Desde la perspectiva funcional se prioriza el tipo de apoyo social recibido por parte de los vínculos sociales con los que cuenta una determinada persona (Cohen y Syme, 1985). Existen cuatro categorías de apoyo social percibido o Enacted (promulgado): emocional (cariño); instrumental (dejarte dinero); informacional (consejos útiles e información para resolver dudas); y appraisal, evaluativo, información proporcionada por los demás útil para la autovaloración y la comparación social (Dunkel-Schetter y Brooks, 2009). La dimensión calidad percibida hace referencia a la evaluación subjetiva por parte del destinatario de la calidad y eficacia del apoyo recibido (Lynch, 1998).

\section{Relaciones entre contextos offline y virtuales y sus implicaciones en el apoyo social}

Actualmente son incipientes las investigaciones sobre la interacción entre los contextos de comunicación online y offline, y sus consecuencias en la vida de las personas. La proximidad física facilita las oportunidades de comunicación y el intercambio de apoyo social online (Ellison, Lampe, Steinfield y Vitak, 2010). El apoyo social se encuentra positivamente relacionado con la interacción social en contextos offline y virtuales (Wang y Wang, 2013), por lo que las posibilidades de complementariedad entre ambos medios son evidentes.

Existe un mayor uso de las redes sociales online para mantener la comunicación por parte de personas que tienen conexiones offline (Quan-Haase e Young, 2010), posibilitándose el mantenimiento del contacto incluso cuando las circunstancias de la vida alejan físicamente unas de las otras (Quan-Haase, Mo y Wellman, 2017). En estudiantes internacionales, las personas de las redes sociales existentes antes de la estancia todavía pueden seguir facilitando apoyo social, lo que demuestra una expansión de las relaciones offline al mundo cibernético (Cemalcilar, Falbo y Stapleton, 2005). En el contexto online los migrantes internacionales pueden 
establecer y mantener redes sociales potencialmente proveedoras de apoyo con familiares, amigos e incluso con extraños, siendo especialmente útil para quienes carecen de apoyo social offline (Chen y Choi, 2011).

La preferencia del uso de las Redes Sociales Virtuales (RSV) en conjunto con el móvil para el reforzamiento de las redes offline se ha evidenciado en inmigrantes adolescentes (Hernández y Alcoceba, 2015) y en adultos mayores (Quan-Haase et al., 2017). En migrantes internacionales el apoyo social mediado por la tecnología es un complemento útil del apoyo social tradicional, con un papel fundamental en la calidad de vida (Chen y Choi, 2011).

\section{Particularidades de los entornos online en la transmisión de apoyo social}

Las redes sociales online tienen una gran capacidad para que se intercambie apoyo social en el caso de jóvenes discapacitados (Suriá, 2017) y entre padres en la crianza de sus hijos (Haslam, Tee y Baker, 2017). Así, las RSV son un medio novedoso por el que el apoyo social puede ser intercambiado (Trepte et al., 2015).

Existen diferencias notables en torno a las bondades del uso de la tecnología e Internet según la naturaleza de la actividad desarrollada. El apoyo social online tiene un impacto positivo en la adaptación sociocultural de los estudiantes internacionales y promueve su satisfacción vital (Wang, Hong y Pi, 2015). En familias trasnacionales las TICs mantienen lazos afectivos y proveen de apoyo emocional a sus miembros (Benítez, 2012). La comunicación por Internet con desconocidos es negativa para el bienestar (Valkenburg y Peter, 2007).

En cuanto a la tipología de apoyo social suministrado de forma preferencial en contextos offline y online existen diferencias relevantes. Las RSV son más adecuadas para el intercambio de apoyo informativo que la comunicación cara a cara, favoreciendo la satisfacción con la vida (Trepte et al., 2015). Se ha comprobado la especial valía de la CMT para la recepción de apoyo informacional en inmigrantes (Chen y Choi, 2011). Las personas con una discapacidad física reciben apoyo emocional e informacional a través de comunidades online (Obst y Stafurik, 2010). Sin embargo, en términos generales, los propios usuarios de Internet perciben más apoyo instrumental y 
emocional en contextos offline; siendo menos importante el tamaño y la heterogeinedad de la red social virtual que en el caso del apoyo informacional (Trepte et al., 2015). En estudiantes migrantes la CMT ayuda a fomentar y mantener los vínculos sociales de su lugar de origen, proporcionándoles apoyo emocional; sin embargo, una sobre-interacción y monopolización del tiempo libre con ellos puede afectar negativamente a su integración en el país de acogida (Lim y Pham, 2016).

Entre los factores que afectan a la búsqueda de apoyo online en los inmigrantes se encuentra el tiempo de residencia, la disponibilidad de apoyo offline y la satisfacción con el apoyo social mediado por la tecnología (Chen y Choi, 2011). El conjunto de disciplinas de lo "social", Trabajo Social, Psicología, Sociología, etc., debe priorizar en su intervención social la alfabetización digital que permita a los inmigrantes sentirse conectados y construir sus propias redes sociales (NASW y ASWB, 2005).

\section{Estudio actual}

Esta investigación tiene como objetivo, atendiendo a las especificidades de las fuentes de procedencia y tipo de apoyo, analizar el efecto de la estructura en términos objetivos de la red de apoyo offline y online sobre la percepción de apoyo social, sentido de comunidad y satisfacción vital de los inmigrantes. Las TICs son potencialmente muy útiles para conservar y fortalecer vínculos con personas, independientemente de la localización. Sin embargo, son incipientes los estudios que han analizado al mismo tiempo el efecto de las redes de apoyo social virtuales y offline en el bienestar y la inclusión de los inmigrantes.

Se tienen en cuenta las redes sociales offline y online tanto en términos cuantitativos, número de personas, como en cualitativos, procedencia y tipo de apoyo suministrado y satisfacción con el apoyo recibido. Se analiza cómo las redes de apoyo offline y online de los inmigrantes de Málaga (España) influyen en su percepción de apoyo social, sentido de comunidad y satisfacción vital. Profundizar en la comprensión de la influencia de las redes sociales sobre el bienestar y la integración de los inmigrantes debe permitir aumentar la efectividad de las futuras intervenciones de trabajadores sociales y de otros profesionales del sector. El análisis comparativo entre el mundo "pre- 
sencial" y el "virtual" posibilita seguir avanzando en el conocimiento de las interacciones que tienen lugar entre dichos ámbitos y, por tanto, contemplar dichos nexos de unión en clave de intervención psicosocial. Así, es necesario analizar la influencia de la estructura de las redes sociales de apoyo desde la perspectiva de las distintas fuentes y los diferentes contextos, tal y cómo indican los modelos ecológicos y sistémicos (Bronfenbrenner, 2005; Levitt, 2005).

\section{METODOLOGÍA UTILIZADA}

\section{Participantes}

En el estudio han participado 1563 inmigrantes residentes en Málaga (España). Málaga es una ciudad multicultural debido a su situación geográfica como puerta de entrada a África y su tradición turística. La inmigración en Málaga se diferencia de otras áreas españolas, existiendo una gran diversidad de procesos migratorios en referencia al origen, motivos de migración o concurrencia de lenguas (Calvo, Asensio y Meléndez-Valdés, 2010). Para cada análisis se utilizó la muestra de participantes que cumplimentó todos los ítems. El 48.3\% varones y el $51.7 \%$ mujeres. El 25.2\% provienen del norte de África, el 27.4\% de América Latina, el 23.6\% de Asia y el $23.8 \%$ de Europa del Este. El 79\% están regularizados, el 5,3\% no regularizados, el 9,6\% están en trámite de regularización y el 6,1\% no contestan. El rango de edad fue de 15 a 80 años $(\mathrm{M}=32.44$, SD =11.44). Esta distribución es representativa de la distribución de inmigrantes de Málaga según los datos del censo de 2017. Según datos del Ayuntamiento de Málaga (2018), a 1 de enero de 2018, la proporción de población extranjera en Málaga en relación al total (573.832 habitantes) es del 6,04\%, y la comunitaria se sitúa en el $1,87 \%$. Con respecto al peso relativo de los distintos países en función de la presencia de cada continente en Málaga, en Europa destacan Ucrania y Rumanía con el 26,68\% y el 16,08\% del total de los extranjeros europeos residentes en Málaga; Marruecos $(69,39 \%)$ y Nigeria $(17,35 \%)$ en África; Paraguay $(28,18 \%)$ y Argentina $(13,44 \%)$ en América; y China $(74,80 \%)$ en Asia.

Para seleccionar a los inmigrantes con acceso a la CMT, se incluyeron únicamente los casos en los que los participantes, para cada 
uno de los tipos de apoyo (emocional, instrumental e informacional), contaban como mínimo con una persona que le diera apoyo offline y online. Mientras que la fuente de apoyo "familia" podía estar constituida por personas situadas en cualquier lugar, las fuentes "amigos españoles" y "amigos inmigrantes" la formaron personas residentes en España. Se entendió por amigo inmigrante a cualquier persona no originaria de España, pudiendo ser o no paisano de los participantes.

\section{Procedimiento}

Esta investigación se desarrolla en el marco del Proyecto I+D (Ref.: PSI2013-40508-P) sobre la integración social y la calidad de vida de la población inmigrante en Málaga. Los participantes fueron voluntarios y se seleccionaron utilizando una metodología de muestreo y encuesta de ruta aleatoria. Se establecieron límites para cada uno de los vecindarios seleccionados y se utilizó un muestreo aleatorio de rutas para designar los bloques, las calles, las aceras, etc. en cada barrio. Estas encuestas fueron recolectadas en la ciudad de Málaga en sus 11 distritos. Los cuestionarios aplicados a las personas que no hablaban español fueron traducidos a su idioma de origen por expertos. Los entrevistadores fueron entrenados para administrar las encuestas. Las encuestas se realizaron en asociaciones, hogares, negocios locales, etc. Una comisión ética oficial (CEUMA) determinó la idoneidad del protocolo en relación al objeto de estudio, al consentimiento informado, la conducta profesional, el procedimiento experimental y los criterios éticos. El instrumento de recogida de datos utilizado en la encuesta se desarrolló a través de la recopilación en un único documento de los cuestionarios de sentido de comunidad, apoyo social (incluyendo el número de personas que proporcionaban apoyo social online y offline para cada una de las fuentes y tipos de apoyo), satisfacción vital y las variables sociodemográficas. Este instrumento de recogida de datos se aplicó de manera integral a cada uno de los participantes en un único encuentro. Los encuentros se realizaron durante los meses de enero, febrero y marzo de 2017 y la duración de los mismos osciló entre los 25 y los 40 minutos. 


\section{Instrumentos}

Apoyo Social: Cuestionario de Frecuencia y Satisfacción con el Apoyo Social (García, Hombrados-Mendieta y Gómez-Jacinto, 2016). Mide la frecuencia y la satisfacción con el apoyo social recibido de la familia, amigos inmigrantes y amigos nativos. El apoyo social se divide en tres áreas: apoyo emocional, apoyo instrumental y apoyo informativo. La frecuencia del apoyo se mide usando una escala Likert (1 = "Rara vez", 5 = "Siempre"). El grado de satisfacción con el apoyo recibido también se mide utilizando una escala Likert (1= "Insatisfecho", 5= "Muy satisfecho"). Es el sumatorio de la frecuencia y satisfacción del apoyo social para cada una de las fuentes. El $\alpha$ de Cronbach se situó en .92 para la familia, .95 para los amigos inmigrantes y .94 para los amigos nativos. Para cada uno de los ítems se requirió que se indicara el número de personas que le proporcionaban cada tipo de apoyo: offline y online (Facebook, Whatsapp, etc.).

Sentido de Comunidad: Índice de Sentido de Comunidad (Chavis, Lee y Acosta, 2008). El SCI- 2 es una medida muy fiable ( $\alpha$ de Cronbach $=.94$ ). Este instrumento se basa en los cuatro componentes del modelo de sentido de comunidad proporcionados por McMillan y Chavis (1986): integración y satisfacción de necesidades, pertenencia al grupo, influencia y conexión emocional compartida. Consta de 24 ítems que se miden a través de una escala tipo Likert: ( $1=$ Nada, 4 $=$ Completamente). El índice global se calculó sumando los 24 ítems ( $\alpha$ de Cronbach $=.94)$.

Satisfacción Vital: Escala de Satisfacción con la Vida (SWLS) (Pavot y Diener, 1993). La SWLS tiene en cuenta que los individuos poseen sus propios criterios y un conjunto de valores que determinan su satisfacción vital. El formato de respuesta es de tipo Likert (1"completamente insatisfecho" a 7 "completamente satisfecho"). El $\alpha$ de Cronbach fue de .90 .

\section{Diseño y análisis de datos}

Se analiza la relación entre las redes de apoyo offline y online de los inmigrantes (número de personas) y la percepción de apoyo social, sentido de comunidad y satisfacción vital. Se realizaron correlaciones no paramétricas de Spearman debido a que las 
variables no cumplieron el supuesto de normalidad. Se compararon todas las correlaciones atendiendo al carácter offline o virtual de las mismas. Para analizar la existencia de diferencias significativas entre las respectivas correlaciones del número de personas que proporcionan apoyo offline o virtual y las variables contempladas se hizo el test $\mathrm{Z}$ de Fisher (http: //vassarstats.net/rdiff.html). Se utilizó un nivel de significación $p<.05$. Al medirse más de 10 observaciones por cada grupo (número de personas de la red de apoyo offline vs número de personas de la red de apoyo online) es viable analizar la existencia de diferencias significativas, aunque sean pruebas no paramétricas, entre las relaciones de ambos grupos (Siegel, 1983). En función del carácter contextualizador del estudio se desarrolló un análisis macro de la realidad malagueña.

\section{RESULTADOS}

En la Tabla 1 se muestra un resumen de los estadísticos descriptivos del número de personas que proporcionan apoyo social. Para las tres fuentes de apoyo analizadas y para los tres tipos de apoyo el número de personas que dan apoyo virtual es ligeramente más elevado que la respectiva cantidad de apoyos offline. Destacan las diferencias entre el número de personas que dan apoyo online y offline para el caso de la familia, especialmente con respecto al apoyo emocional $(M=12.41, D T=65.51$ para el número de personas que proporciona el apoyo virtualmente; $M=5.23, D T=23.29$ para el número de personas que proporcionan el apoyo offline). En una comparativa entre los tres tipos de fuente se observa como respectivamente para cada tipo de apoyo y contexto (offline/online) el número de personas que proporcionan un determinado apoyo en términos generales es bastante similar en las tres fuentes; salvo para el caso del apoyo emocional virtual de la familia, donde el número de personas que proporcionan dicho apoyo es considerablemente superior que para los casos de los amigos españoles e inmigrantes. La $M$ de personas, para las tres fuentes, que proporcionan apoyo emocional virtual es la más elevada, mientras que la más baja corresponde al apoyo instrumental offline (especialmente para la familia $M=3.86, D T=2.99$ ). 


\section{TABLA 1}

\section{RESÚMENES ESTADÍSTICOS DESCRIPTIVOS DEL NÚMERO DE PERSONAS QUE PROPORCIONAN APOYO SOCIAL}

\begin{tabular}{|c|c|c|c|c|c|}
\hline Fuente & Tipo & $\begin{array}{c}\text { Número de } \\
\text { personas } \\
\text { Offline/ } \\
\text { Online }\end{array}$ & $\mathrm{N}$ & Media & DT \\
\hline \multirow[t]{6}{*}{ Familia } & Emocional & Offline & 1563 & 5.23 & 23.29 \\
\hline & & Online & 1245 & 12.41 & 65.51 \\
\hline & Instrumental & Offline & 1508 & 3.86 & 2.99 \\
\hline & & Online & 1006 & 6.50 & 26.44 \\
\hline & Informacional & Offline & 1495 & 4.63 & 20.37 \\
\hline & & Online & 1115 & 6.80 & 25.75 \\
\hline \multirow[t]{6}{*}{ Amigos españoles } & Emocional & Offline & 1512 & 5.82 & 28.47 \\
\hline & & Online & 1024 & 7.83 & 35.57 \\
\hline & Instrumental & Offline & 1418 & 5.31 & 29.27 \\
\hline & & Online & 872 & 6.43 & 27.31 \\
\hline & Informacional & Offline & 1464 & 5.77 & 28.86 \\
\hline & & Online & 991 & 6.83 & 26.26 \\
\hline \multirow[t]{6}{*}{ Amigos inmigrantes } & Emocional & Offline & 1394 & 6.58 & 41.58 \\
\hline & & Online & 975 & 9.24 & 50.24 \\
\hline & Instrumental & Offline & 1323 & 4.46 & 21.55 \\
\hline & & Online & 852 & 5.29 & 8.39 \\
\hline & Informacional & Offline & 1358 & 4.85 & 21.67 \\
\hline & & Online & 944 & 5.71 & 10.18 \\
\hline
\end{tabular}

Fuente: elaboración propia (2018).

La Tabla 2 presenta las correlaciones entre la frecuencia y satisfacción con los diferentes tipos de apoyo y el número de personas que proporcionan el apoyo de manera offline y virtual. Los análisis, a través del coeficiente de correlación de Spearman $(R h o)$, señalaron, para todas las fuentes y tipologías de apoyo, una relación significativa y positiva entre el número de personas que brindan apoyo de manera offline y online y la respectiva frecuencia 
y satisfacción con el determinado apoyo recibido. La correlación más débil, aunque significativa, fue la relación entre la satisfacción con el apoyo instrumental de la familia y el número de familiares que dan ese apoyo virtualmente $(R h o=.077, p<.05)$. En todos los casos, comparativamente, la relación es más fuerte, y de igual signo, para el contacto offline que para el virtual (número de personas que proporcionan el respectivo apoyo offline en relación al número de personas que brindan ese apoyo de manera virtual). El resultado del Fisher's Z test señaló que existían diferencias significativas entre las respectivas correlaciones del número de personas que proporcionan apoyo offline y virtual en los siguientes casos: en la satisfacción con el apoyo instrumental proveniente de la familia $(Z=2.22, p=.0264)$, en la frecuencia del apoyo emocional por parte de los amigos españoles $(Z=2.13, p=.0332)$ y con respecto a los amigos inmigrantes tanto en la frecuencia $(Z=2.11, p=.0349)$ como en la satisfacción del apoyo emocional $(Z=2.1, p=.0357)$.

En relación a las fuentes analizadas, las correlaciones más fuertes se desarrollan en el ámbito de los amigos españoles e inmigrantes, mientras que las correlaciones de los apoyos de la familia y el número de personas offline y virtuales que dan ese apoyo son más débiles (En la relación entre la satisfacción con el apoyo emocional recibido y el número de personas que de manera offline proporcional ese apoyo: familia (Rho=.153, $p<.01)$, amigos españoles (Rho=.310, $p<.01)$, amigos inmigrantes $(R h o=.274, p<.01))$. En términos generales las respectivas correlaciones de la frecuencia y la satisfacción con el apoyo con el número de personas que proporcionan el apoyo de manera offline y virtual son de una magnitud similar; siendo especialmente análogas para el contacto offline (Para el apoyo instrumental de la familia: frecuencia $(R h o=.165, p<.01)$, satisfacción $(R h o=.165, p<.01)$. El Fisher's Z test evidenció que en ningún caso existían diferencias significativas, tanto en el plano offline como en el virtual, en las respectivas correlaciones de la frecuencia y la satisfacción con el apoyo y el número de personas que proporcionan dicho apoyo.

Con respecto a las diversas tipologías de apoyo la magnitud de las correlaciones entre el número de personas que dan apoyo offline y virtual y la frecuencia y satisfacción con un determinado apoyo varía ligeramente en función de la procedencia del mismo (Fuente). En relación a la familia destaca la magnitud del apoyo informacional (Respecto al apoyo offline $R h o=.202$, $p<.01$ para la 
frecuencia y $R h o=.199, p<.01$ para la satisfacción con el apoyo; para el apoyo virtual $R h o=.154, p<.01$ para la frecuencia y $R h o=.137$, $p<.01$ para la satisfacción con el apoyo). En el caso de los amigos españoles sobresalen las relaciones con respecto al apoyo emocional e instrumental; especialmente en referencia al apoyo emocional offline ( $R h o=.363, p<.01$ para la frecuencia y $R h o=.310, p<.01$ para la satisfacción con el apoyo).

\section{TABLA 2}

CORRELACIONES ENTRE LA FRECUENCIA Y SATISFACCIÓN CON LAS DIFERENTES TIPOLOGÍAS DE APOYO Y EL NÚMERO DE PERSONAS QUE DAN EL DETERMINADO APOYO DE MANERA OFFLINE Y ONLINE

\begin{tabular}{|c|c|c|c|c|}
\hline Fuente & Tipo & $\mathrm{F} / \mathrm{S}$ & Offline & Online \\
\hline \multirow[t]{6}{*}{ Familia } & Emocional & Frecuencia & $.155^{\star \star}$ & $.119^{\star \star}$ \\
\hline & & Satisfacción & $.153^{\star \star}$ & $.102^{\star \star}$ \\
\hline & Instrumental & Frecuencia & $.165^{\star \star}$ & $.114^{\star \star}$ \\
\hline & & Satisfacción & $.166^{\star \star}$ & $.077^{\star}$ \\
\hline & Informacional & Frecuencia & $.202^{\star \star}$ & $.154^{\star \star}$ \\
\hline & & Satisfacción & $.199^{\star \star}$ & $.137^{\star \star}$ \\
\hline \multirow[t]{6}{*}{ Amigos españoles } & Emocional & Frecuencia & $.363^{\star \star}$ & $.286^{\star \star}$ \\
\hline & & Satisfacción & $.310^{\star \star}$ & $.245^{\star \star}$ \\
\hline & Instrumental & Frecuencia & $.343^{\star \star}$ & $.286^{\star \star}$ \\
\hline & & Satisfacción & $.307^{\star \star}$ & $.255^{\star \star}$ \\
\hline & Informacional & Frecuencia & $.308^{\star \star}$ & $.237^{\star \star}$ \\
\hline & & Satisfacción & $.292^{\star \star}$ & $.225^{\star \star}$ \\
\hline \multirow[t]{6}{*}{ Amigos inmigrantes } & Emocional & Frecuencia & $.301^{\star \star}$ & $.219^{\star \star}$ \\
\hline & & Satisfacción & $.274^{\star \star}$ & $.191^{\star \star}$ \\
\hline & Instrumental & Frecuencia & $.311^{\star *}$ & $.239^{\star \star}$ \\
\hline & & Satisfacción & $.249^{\star \star}$ & $.194^{\star \star}$ \\
\hline & Informacional & Frecuencia & $.293^{\star \star}$ & $.220^{\star}$ \\
\hline & & Satisfacción & $.255^{\star \star}$ & $.187^{\star \star}$ \\
\hline
\end{tabular}

${ }^{*} p<.05$

${ }^{\star *} \mathrm{p}<.01$

Fuente: elaboración propia (2018). 
En la Tabla 3 se muestran las relaciones entre el número de personas que proporcionan un determinado tipo de apoyo y la satisfacción vital y el sentido de comunidad. Los análisis realizados, a través del coeficiente de correlación de Spearman (Rho), en términos generales evidenciaron una relación significativa y positiva entre el número de personas que proporcionaban el respectivo apoyo, tanto offline como online, y la satisfacción vital y el sentido de comunidad. De entre todas las relaciones analizadas únicamente cuatro correlaciones en torno a los amigos inmigrantes no resultaron significativas, concretamente: la relación entre el número de personas que proporcionan apoyo emocional virtual y la satisfacción vital ( $R h o=.055, p>.05)$, la correlación entre el número de personas que proporcionan apoyo instrumental virtual tanto con la satisfacción vital (Rho=.066, $p>.05)$ como con el sentido de comunidad $(R h o=.038, p>.05)$ y la relación entre el número de personas que proporcionan apoyo informacional virtual y la satisfacción vital ( $R h o=.063, p>.05)$. Además, de manera general, comparativamente, la correlación tanto con la satisfacción vital como con el sentido de comunidad fue de mayor magnitud, aunque de igual signo, para el apoyo offline en detrimento del apoyo virtual. Así, únicamente dos relaciones, insertas en la fuente "familia", fueron más fuertes en el plano virtual que en el offline, concretamente: la relación entre el número de familiares que proporcionan apoyo instrumental virtual y el sentido de comunidad ( $R h o=.092, p<.01)$ y la correlación entre la cantidad de familiares que proporcionan apoyo informacional virtual y el sentido de comunidad ( $R h o=.105, p<.01)$. El test $\mathrm{Z}$ de Fisher indicó que únicamente existía una diferencia significativa entre las respectivas correlaciones del número de personas que proporcionan apoyo offline y online y las variables analizadas (satisfacción vital y sentido de comunidad). Concretamente el par de correlaciones en el apoyo instrumental de la familia y la satisfacción vital, en la que la correlación offline, siguiendo la tendencia general, fue más fuerte $(Z=2.33, p=.0198)$. 


\section{TABLA 3}

CORRELACIONES ENTRE NÚMERO DE PERSONAS QUE PROPORCIONAN APOYO Y LA SATISFACCIÓN VITAL Y EL SENTIDO DE COMUNIDAD

\begin{tabular}{|c|c|c|c|c|}
\hline Fuente & Tipo & Offline/Online & SV & SC \\
\hline \multirow[t]{6}{*}{ Familia } & Emocional & Offline & $.186^{\star \star}$ & $.125^{\star \star}$ \\
\hline & & Online & $.127^{\star \star}$ & $.107^{\star \star}$ \\
\hline & Instrumental & Offline & $.183^{\star \star}$ & $.086^{\star \star}$ \\
\hline & & Online & $.090^{\star *}$ & $.092^{\star *}$ \\
\hline & Informacional & Offline & $.184^{\star \star}$ & $.097^{\star \star}$ \\
\hline & & Online & $.108^{\star \star}$ & $.105^{\star \star}$ \\
\hline \multirow[t]{6}{*}{ Amigos españoles } & Emocional & Offline & $.215^{\star \star}$ & $.157^{\star \star}$ \\
\hline & & Online & $.147^{\star \star}$ & $.101^{\star *}$ \\
\hline & Instrumental & Offline & $.207^{\star \star}$ & $.155^{\star \star}$ \\
\hline & & Online & $.151^{\star \star}$ & $.124^{\star \star}$ \\
\hline & Informacional & Offline & $.228^{\star \star}$ & $.162^{\star \star}$ \\
\hline & & Online & $.152^{\star \star}$ & $.112^{\star \star}$ \\
\hline \multirow[t]{6}{*}{ Amigos inmigrantes } & Emocional & Offline & $.080^{\star \star}$ & $.097^{\star \star}$ \\
\hline & & Online & .055 & $.071^{*}$ \\
\hline & Instrumental & Offline & $.108^{\star \star}$ & $.099^{\star *}$ \\
\hline & & Online & .066 & .038 \\
\hline & Informacional & Offline & $.089^{\star \star}$ & $.126^{\star \star}$ \\
\hline & & Online & .063 & $.096^{\star \star}$ \\
\hline
\end{tabular}

${ }^{*} \mathrm{p}<.05$

${ }^{\star *} \mathrm{p}<.01$

Fuente: elaboración propia (2018).

En referencia a las fuentes analizadas generalmente las correlaciones más intensas se sitúan en torno a los amigos españoles $(R h o=.228, p<.01$, para la correlación entre el número de personas que le proporcionan apoyo instrumental offline y la satisfacción vital), seguido de las relaciones en el ámbito de la familia ( $R h o=.186$, $p<.01$, para la correlación entre el número de familiares que le proporcionan apoyo emocional offline y la satisfacción vital). A nivel global las relaciones más débiles se enmarcan dentro de la fuente 
“amigos inmigrantes", siendo la de mayor magnitud la correlación entre el número de amigos inmigrantes que le proveen de apoyo informacional offline y el sentido de comunidad (Rho=.126, $p<.01)$. Con respecto a los diferentes tipos de apoyo contemplados (emocional, instrumental e informacional), se aprecia como a nivel general la magnitud de las correlaciones entre el número de apoyos offline y virtuales y la satisfacción vital y el sentido de comunidad son similares para una misma fuente de procedencia. Se observa cierta tendencia a la semejanza de la magnitud de las respectivas correlaciones, en términos comparativos para las mismas relaciones analizadas, del sentido de comunidad y la satisfacción vital. El test $\mathrm{Z}$ de Fisher señaló que de todas las correlaciones estudiadas únicamente en dos casos existía una diferencia significativa entre las respectivas correlaciones entre el número de personas que proporcionan un determinado apoyo y la satisfacción vital y el sentido de comunidad (Par de correlaciones entre el número de personas que dan apoyo instrumental offline de la familia y la satisfacción vital/sentido de comunidad $Z=2.71, p=.0067$; par de relaciones entre el número de familiares que dan apoyo informacional offline y la satisfacción vital $/$ sentido de comunidad $Z=2.43, p=.0151$ ).

\section{DISCUSIÓN}

La era tecnológica actual ha supuesto un cambio notorio en la forma de comunicarse, y de relacionarse, de las personas equiparable al desarrollo de la imprenta. Las condiciones especiales que se le presuponen a los inmigrantes, debido tanto a las frecuentes situaciones de trasnacionalismo como a la necesidad de crear redes de apoyo, posicionan a este colectivo en un lugar predilecto para analizar la influencia de la tecnología e Internet en sus vidas (Codagnone y Kluzer, 2011; Peng, 2016). En palabras de González et al. (2015) es evidente que los ámbitos de la comunicación y el social se funden incluso terminológicamente, siendo cuanto menos llamativo que una de las herramientas de comunicación con más impacto en la historia, las redes sociales virtuales, sean conocidas usualmente como "redes sociales"; término también referido al conjunto de individuos que interaccionan sin necesidad de mediación tecnológica. 
Este estudio, atendiendo a las especificidades de las fuentes de procedencia y el tipo de apoyo, ha evidenciado resultados interesantes sobre la influencia de la estructura de la red en la percepción de apoyo social, sentido de comunidad y satisfacción vital de los inmigrantes. Los resultados mostraron que el número de personas que dan apoyo de manera online para las tres fuentes, especialmente familia, y para los tres tipos de apoyo es comparativamente ligeramente superior que para el caso de los apoyos offline. Las limitaciones de la proximidad física por parte de los integrantes de la familia, dado que un considerable número de miembros puede residir en el lugar de origen, podrían explicar la especial conectividad virtual con dicho colectivo. Estos resultados apoyan la validez de los fundamentos teóricos que señalan el éxito de la CMT para conservar el contacto con familiares y amigos, al posibilitar incluso una cierta presencia en las comunidades de origen y suponer la ruptura del límite espacio-temporal humano (Francisco, 2015). La hipótesis del cerebro social ha sugerido que el tamaño de la red social tiene una magnitud característica en los seres humanos determinada por limitaciones cognitivas y por los costes temporales de las relaciones de servicio (Dunbar, 1998). Los resultados de esta investigación están en consonancia con estudios que han demostrado el potencial de las redes sociales online para reducir los costes temporales de las relaciones sociales y propiciar el mantenimiento de redes sociales mucho más grandes (Dunbar, 2016).

En las tres fuentes analizadas (familia, amigos españoles y amigos inmigrantes) se aprecia como comparativamente para cada tipo de apoyo y contexto (offline/online), el número de personas que proporcionan un determinado apoyo generalmente es bastante similar en las tres fuentes; salvo en el caso del apoyo emocional virtual de la familia que es considerablemente superior. Estos hallazgos confirman por un lado que los inmigrantes en Málaga tienden a construir redes de apoyo multiorigen pero normalmente uniformes $\mathrm{y}$, por otro, que la distancia geográfica, que preferentemente se le presupone a la familia, es un elemento clave para que los inmigrantes se decanten por contactos virtuales. Los resultados obtenidos en torno a la estructura de apoyo de la familia son coherentes con otras investigaciones que señalan la existencia de un mayor uso de las RSV para mantener la comunicación por parte de personas que tienen conexiones offline; al posibilitar su contacto incluso cuando las circunstancias de la vida impiden su encuentro físico (Ellison, 
Steinfield y Lampe, 2007; Quan-Haase et al., 2017). La red de personas que proporcionan apoyo emocional online es la más elevada para todas las fuentes, mientras que la más reducida corresponde al apoyo instrumental offline, especialmente de la familia. Esto es una evidencia de que las RSV son un medio por el que las personas inmigrantes pueden intercambiar apoyo social de manera eficiente y de la existencia de una mayor percepción de apoyo instrumental en contextos offline (Trepte et al., 2015).

Para todas las fuentes, contextos y tipologías de apoyo hubo una correlación significativa y positiva entre el número de personas que le brindaban ese apoyo y la frecuencia y la satisfacción con el apoyo recibido. Una estructura amplia de la red de apoyo es importante de cara a la valoración positiva de la misma por parte de los inmigrantes residentes en Málaga (Şerban, 2007). Los resultados en el ámbito virtual pueden estar explicados por el hecho de que todas las fuentes están compuestas por personas con las que han tenido una relación previa, previsiblemente cercanas, elemento que la literatura académica ha señalado clave para que la comunicación mediada por la tecnología tenga una potencial influencia positiva en el bienestar (Valkenburg y Peter, 2007). Los hallazgos apoyan investigaciones previas que defienden la funcionalidad de la CMT durante los procesos de aculturación al facilitar el contacto entre grupos con orígenes etnoculturales diferenciados, siempre que se utilice como complemento de socialización en la cultura de acogida (Cemalcilar et al., 2005; Retortillo y Rodríguez, 2010). El hecho de que todas las relaciones, tanto en el plano offline como en el online, sean significativas y positivas apoya la teoría de que la CMT complementa en lugar de reemplazar el apoyo offline de los inmigrantes (Chen y Choi, 2011). Además, tanto la relativa similitud en el número de personas que proporcionan un determinado apoyo en el contexto offline y en el online, como el alto grado de cercanía de las fuentes analizadas pueden entenderse como un indicio de que en la mayoría de ocasiones, cuando la proximidad física lo permite, una misma persona proporciona apoyo en ambos contextos. Este planteamiento está en sintonía con la hipótesis de la eficiencia de Nie et al. (2002), la cual señala que Internet posibilita nuevas opciones para mantener, complementar y crear redes sociales. Las RSV no van a suponer un reemplazo de las formas tradicionales de comunicación, sino que ambas tienden a desarrollar una relación de complementariedad. La proximidad física facilita las oportunidades de comunicación y el intercambio de apoyo social 
online (Ellison et al., 2010). Los resultados reafirman la concepción teórica que señala que el apoyo social se encuentra positivamente relacionado con los vínculos de interacción social en contextos tanto offline como online (Wang y Wang, 2013). Estos hallazgos refuerzan la teoría de que el desarrollo tecnológico ha fomentado una conexión virtual permanente que posibilita que la vivencia del mundo online no sea una experiencia puntual sino un contexto simultáneo y complementario en la vida de las personas (González et al., 2015).

En todos los casos, comparativamente, la relación positiva entre el número de personas que proporcionan apoyo y la frecuencia y satisfacción con el apoyo es más fuerte para el contexto offline que para el online. Esto podría entenderse como una evidencia de la gran importancia que tiene la construcción de una red de apoyo offline sólida, y por tanto del contacto físico, por parte de los inmigrantes para valorar de manera positiva el apoyo social percibido. Estos resultados podrían sugerir que la red de apoyo offline en términos cuantitativos (cantidad) tiene una influencia mayor que la virtual en la percepción de apoyo por parte de las personas inmigrantes; pudiéndose inferir que desde esta perspectiva la red de apoyo offline es más eficiente. Si bien los hallazgos de esta investigación refuerzan la importancia de un tamaño amplio de la red virtual de cara a una percepción positiva de apoyo social (Eastin y LaRose, 2005; Lee et al., 2011; Trepte et al., 2015). La existencia de apoyo social depende en primera instancia del tamaño y la calidad de las redes sociales, tanto el contexto como la fuente, que provee de oportunidades a un individuo para interactuar con otros (Wong et al., 2017).

Las correlaciones más fuertes entre el número de personas que proporcionan un determinado apoyo y la frecuencia y satisfacción con el apoyo se desarrollan en el ámbito de los amigos españoles seguido de los amigos inmigrantes, mientras que las más débiles son las relacionadas con la familia. Los resultados referentes a los amigos españoles reflejan la importancia que tiene la consolidación de una red amplia de amigos autóctonos de cara a la percepción positiva de apoyo social por parte del colectivo de inmigrantes. Sin embargo, la percepción positiva de apoyo por parte de la familia no está tan relacionada con el número de miembros que le proporcionan dicho apoyo, pudiendo deberse a que la aportación de cada miembro de la familia a la percepción positiva del apoyo recibido puede ser potencialmente elevada y específica atendiendo a las características de cada contexto. Estos resultados apoyarían la especial importancia 
de cada uno de los miembros de la familia en la provisión de apoyo instrumental, emocional e informacional (Chib et al., 2013).

Las similitudes en las magnitudes de las correlaciones referentes a la frecuencia y a la satisfacción con el apoyo y el número de personas que proporcionan un determinado tipo de apoyo, dentro de un contexto y una fuente determinada, evidencian tanto la importancia en términos cuantitativos de la estructura de apoyo como el carácter diferencial de la naturaleza, e incluso de las necesidades percibidas, de las distintas fuentes de apoyo analizadas. Las semejanzas en la intensidad de las relaciones de estas tres variables (número de personas que proporcionan apoyo, frecuencia y satisfacción con el apoyo) ponen de manifiesto la relevancia de la estructura, incluyendo la perspectiva cuantitativa, de las redes de apoyo objetivas de los inmigrantes a la hora de explicar la satisfacción con los diversos apoyos recibidos. Esto está en consonancia con el planteamiento de Thoits (1982), al demostrarse que la cantidad de apoyo social recibido, además del tipo y la procedencia del mismo, son elementos fundamentales para la valoración positiva del apoyo social percibido.

La robusta relación positiva evidenciada entre el número de personas que proporcionaban apoyo social, offline y online, y el sentido de comunidad y la satisfacción vital ratifica tanto que el sentido de comunidad y la satisfacción vital son dos constructos especialmente interrelacionados en personas inmigrantes (Millán-Franco, GómezJacinto, Hombrados-Mendieta, García-Martín y García-Cid, 2019), como la relevancia en términos numéricos de la red de apoyo para explicar su bienestar e integración social (Trepte et al., 2015; Wong et al., 2017). Así, se aprecia cierta similitud en la magnitud de las respectivas correlaciones lo cual evidencia que la estructura de las redes de apoyo, en términos cuantitativos, ejerce una influencia bastante similar en ambas variables. La mayor intensidad de las correlaciones con apoyo offline puede considerarse como una evidencia de que la red de apoyo presencial tiene una mayor capacidad para influir positivamente en los niveles de sentido de comunidad y satisfacción vital. Se confirman los beneficios de la comunicación mediada por la tecnología para mantener el contacto con la familia y amigos que comparten área de residencia y para desarrollar vínculos a través de intercambios de apoyo social con los miembros de las comunidades en las que participan los inmigrantes. Esto contribuye positivamente al desarrollo de satisfacción vital y a la construcción de sentido 
de comunidad hacia el lugar donde desarrollan sus vidas (Castro y González, 2008; Francisco, 2015; Viruela, 2007). En las fuentes de procedencia del apoyo, las correlaciones más fuertes se sitúan en el plano de los amigos españoles, seguidos de los miembros de la familia. Estos resultados evidencian la especial importancia, en términos de satisfacción vital y sentido de comunidad, que supone para las personas inmigrantes contar con una red amplia de apoyo por parte de autóctonos para su integración y bienestar (Domínguez-Fuentes y Hombrados-Mendieta, 2012; Millán-Franco, Gómez-Jacinto, Hombrados-Mendieta, González-Castro y García-Cid, 2019).

Los resultados de este estudio deben interpretarse considerando algunas limitaciones. El diseño transversal menoscaba las conclusiones de causalidad sobre las relaciones entre variables (León y Montero, 2003), si bien la fundamentación teórica desarrollada a lo largo de este documento permite asumir las relaciones planteadas entre las variables. Por otro lado, hubiera sido positivo atender a las particularidades de procedencia de los inmigrantes, si bien dado el carácter exploratorio y la orientación contextualizadora de esta investigación se optó por un análisis macro de la realidad malagueña.

\section{CONCLUSIONES}

A lo largo de este estudio se ha evidenciado el beneficio que supone para las personas inmigrantes disponer de una red de apoyo amplia y heterogénea, posicionándose como un elemento de gran valía en términos de percepción de apoyo social, sentido de comunidad y satisfacción vital. Los resultados han sugerido que la red de apoyo offline en términos objetivos favorece más el desarrollo de las variables de adaptación psicosocial contempladas. Estos hallazgos tienen implicaciones importantes para los responsables políticos involucrados en la provisión de servicios apropiados para los inmigrantes, concluyendo, a su vez, que los profesionales de los Servicios Sociales, trabajadores/as sociales, psicólogos/ as, sociólogos/as y profesiones afines, deben integrar de forma prioritaria en sus funciones laborales la enseñanza del dominio de la tecnología para aumentar en los usuarios las posibilidades de construcción de redes de apoyo y de desarrollo de sentido de comunidad y satisfacción vital. 


\section{REFERENCIAS}

Álvarez de Sotomayor, A. (2008). El papel de las redes étnicas en el logro educativo de los hijos de los inmigrantes ¿recursos u obstáculos? Migraciones, 23, 45-77.

Angner, E. (2010). Subjective well-being. The Journal of Socio-Economics, 39, 361-368. https://doi.org/10.1016/j.socec.2009.12.001

Ayuntamiento de Málaga (2018). Estadísticas demográficas extranjeros 2018. http:/gestrisam.malaga.eu/export/sites/default/economia/gestrisam/ portal/menu/seccion_0006/documentos/2018_Estadxstica_Extranjeros_ Mxlaga.pdf

Benítez, J. L. (2012). Salvadoran transnational families: ICT and communication practices in the network society. Journal of Ethnic and Migration Studies, 38(9), 1439-1449. https://doi.org/10.1080/136918 3X.2012.698214

Bronfenbrenner, U. (2005). Making human beings human: Bioecological perspectives on human development. Thousand Oaks, CA: Sage.

Calvo, A., Asensio, M. A., y Meléndez-Valdés, M. (2010). La inmigración en el municipio de Málaga. Asociación pluralismo, conciencia y derecho, PLUCONDE. Valencia: Tirant lo Blanch.

Castro, L. A., y González, V. M. (2008). Being part of the life of one's hometown: Strategies to support community connectedness. PsychNology Journal 6(1), 61-83.

Cemalcilar, Z., Falbo, T., y Stapleton, L. M. (2005). Cyber communication: A new opportunity for international students' adaptation? International Journal of Intercultural Relations, 29(1), 91-110. https://doi.org/10.1016/j. ijintrel.2005.04.002

Chavis, D. M., Lee, K. S., y Acosta, J. D. (2008). The Sense of Community (SCI) Revised: The Reliability and Validity of the SCI-2. Paper presented at the $2^{\text {nd }}$ International Community Psychology Conference, Lisboa, Portugal.

Chen, W., y Choi, A. S. K. (2011). Internet and social support among Chinese migrants in Singapore. New Media \& Society, 13(7), 1067-1084. https:// doi.org/10.1177/1461444810396311

Chib, A., Wilkin, H. A., y Hua, S. R. M. (2013). International migrant workers' use of mobile phones to seek social support in Singapore. Information Technologies \& International Development, 9(4), 19-34.

Codagnone, C., y Kluzer, S. (2011). ICT for the Social and Economic Integration of Migrants into Europe. European Commission. Institute for Prospective Technological Studies. Recuperado de http://ftp.jrc.es/ EURdoc/JRC63183.pdf

Cohen, S., y Syme, L. (1985). Social support and health. New York: Academic Press. 
Comeforo, K. (2016). Confirming the PSOC through discourse: Toward a grounded theory of discursive sense of community (DSOC). Journal of Community Psychology, 44(5), 620-637. https://doi.org/10.1002/ jcop. 21790

De Miguel Luken, V., y Tranmer, M. (2010). Personal support networks of immigrants to Spain: A multilevel analysis. Social Networks, 32(4), 253262. http://dx.doi.org/10.1016/j.socnet.2010.03.002

Diminescu, D. (2008). The connected migrant: An epistemological manifesto. Social Science Information, 47(4), 565-579. https://doi. org/10.1177/0539018408096447

Domínguez-Fuentes, J. M., y Hombrados-Mendieta, M. I. (2012). Social support and happiness in immigrant women in Spain. Psychological Reports, 110(3), 977-990. https://doi.org/10.2466/17.02.20.21.PR0.110.3.977-990

Dunbar, R. I. M. (1998). The social brain hypothesis. Evolutionary Anthropology: Issues, News, and Reviews, 6(5), 178-190. https://doi. org/10.1002/(SICI)1520-6505(1998)6:5<178::AID-EVAN5>3.0.CO;2-8

Dunbar, R. I. M. (2016). Do online social media cut through the constraints that limit the size of offline social networks? Royal Society Open Science, 3, 150292. https://doi.org/10.1098/rsos.150292

Dunkel-Schetter, C., y Brooks, K. P. (2009). Social support, nature of. In H. T. Reis y S. Sprecher (Eds.), Encyclopedia of human relationships (pp. 1565-1570). Thousand Oaks, CA: SAGE Publications, Inc. h t t p : / / dx.doi.org/10.4135/9781412958479.n515

Eastin, M. S., y LaRose, R. (2005). Alt.support: Modeling social support online. Computers in Human Behavior, 21(6), 977-992. h t t p : / / dx.doi.org/10.1016/j.chb.2004.02.024

Ellison, N., Lampe, C., Steinfield, C, y Vitak, J. (2010). With a little help from my friends: How social network sites affect social capital processes. In Papacharissi, Z. (Ed.), The networked self: Identity, community, and culture on social network sites (pp. 124-145). New York: Routledge.

Ellison, N., Steinfield, C., y Lampe, C. (2007). The benefits of facebook "Friends:" social capital and college students' use of online social network sites. Journal of Computer-Mediated Communication, 12(4), 1143-1168. https://doi.org/10.1111/j.1083-6101.2007.00367.x

Ferrer, R., Palacio, J., Hoyos, O., y Madariaga, C. (2014). Proceso de aculturación y adaptación del inmigrante: características individuales y redes sociales. Psicología Desde El Caribe, 31(3), 557-576. https://doi. org/10.14482/psdc.31.3.4766

Francisco, V. (2015). 'The internet is magic': Technology, intimacy and transnational families. Critical Sociology, 41(1), 173-190. https://doi. org/10.1177/0896920513484602

García, M. A., Hombrados-Mendieta, I., y Gómez-Jacinto, L. (2016). A Multidimensional Approach to Social Support: The Questionnaire on the Frequency of and Satisfaction with Social Support (QFSSS). Anales de 
Psicología, 32(2), 501-515. https://doi.org/10.6018/analesps.32.2.201941

Garcini, L. M., Murray, K. E., Zhou, A., Klonoff, E. A., Myers, M. G., y Elder,

P. (2016). Mental health of undocumented immigrant adults in the

United States: A systematic review of methodology and findings. Journal of Immigrant \& Refugee Studies, 14(1), 1-25. https://doi.org/10.1080/155 62948.2014 .998849

González, S., Zayas, A., y Guil, R. (2015). Relaciones personales en la sociedad de las redes sociales virtuales. RESED, 3, 34-49.

Guruge, S., Thomson, M. S., George, U., y Chaze, F. (2015). Social support, social conflict, and immigrant women's mental health in a Canadian context: A scoping review. Journal of Psychiatric and Mental Health Nursing, 22, 655-667. https://doi.org/10.1111/jpm.12216

Haslam, D. M., Tee, A., y Baker, S. (2017). The use of social media as a mechanism of social support in parents. Journal of Child and Family Studies, 26(7), 2026-2037. http://dx.doi.org/10.1007/s10826-017-0716-6

Hernández, C., y Alcoceba, J. A. (2015). Socialización virtual, multiculturalidad y riesgos de los adolescentes latinoamericanos en España. Icono 14 (13), 116-141. https://doi.org/10.7195/ri14.v13i2.787

Lee, G., Lee, J., y Kwon, S. (2011). Use of social-networking sites and subjective well-being: A study in South Korea. Cyberpsychology, Behavior, and Social Networking, 14(3), 151-155. https://doi.org/10.1089/ cyber.2009.0382

León, O. G., y Montero, I. (2003). Métodos de investigación en Psicología y Educación ( $3^{\mathrm{a}}$ ed.). Madrid: McGrawHill.

Levitt, M. J. (2005). Social relations in childhood and adolescence: The convoy model perspective. Human Development, 48, 28-47. https://doi. org/10.1159/000083214

Lim, S. S., y Pham, B. (2016). 'If you are a foreigner in a foreign country, you stick together': Technologically mediated communication and acculturation of migrant students. New Media \& Society, 18(10), 21712188. https://doi.org/10.1177/1461444816655612

Lin, N. (1986). Conceptualizing social support. In N. Lin, A. Dean, y W. M. Ensel (Eds.), Social support, life events and depression (pp. 17-30). Londres: Academic Press.

Lin, N., y Ensel, W. M. (1989). Life stress and health: Stressors and resources. American Sociological Review, 54(3), 382-399.

Llopis, J. (2005). Redes sociales y Apoyo Social. Una aproximación a los Grupos de Autoayuda. Perifèria, 3, 1-24.

Lynch, S. A. (1998). Who supports whom? how age and gender affect the perceived quality of support from family and friends. The Gerontologist, 38(2), 231-238. https://doi.org/10.1093/geront/38.2.231

Madianou, M., y Miller, D. (2013). Polymedia: Towards a new theory of digital media in interpersonal communication. International Journal of Cultural Studies, 16(2), 169-187. https://doi.org/10.1177/1367877912452486 
Martínez de Ibarreta, C., Redondo, R., y Rúa, A. (2011). Impacto de las redes sociales de apoyo sobre la vulnerabilidad de los inmigrantes. Un análisis del caso español. Migraciones, 29, 61-94.

Maya, I. (2004). Sentido de comunidad y potenciación comunitaria. Apuntes de Psicología, 22 (2), 187-211.

McMillan, D. W., y Chavis, D. M. (1986). Sense of community: A definition and theory. Journal of Community Psychology, 14, 6-23.

Millán-Franco, M., Gómez-Jacinto, L., Hombrados-Mendieta, I., GarcíaMartín, M. A., y García-Cid, A. (2019). Influence of time of residence on the sense of community and satisfaction with life in immigrants in Spain: The moderating effects of sociodemographic characteristics. Journal of Community Psychology, 47, 1078-1094. https://doi.org/10.1002/ jcop. 22172

Millán-Franco, M., Gómez-Jacinto, L., Hombrados-Mendieta, I., GonzálezCastro, F., y García-Cid, A. (2019). The effect of length of residence and geographical origin on the social inclusion of immigrants. Psychosocial Intervention. Ahead of print. https://doi.org/10.5093/pi2019a10

National Association of Social Workers (NASW) y Association of Social Work Boards (ASWB). (2005). NASW and ASWB standards for technology and social work practice. Washington, DC: Authors.

Nie, N. H., Hillygus, D. S., y Erbring, L. (2002). Internet use, interpersonal relations, and sociability: A time diary study. In B. Wellman y C. Haythornthwaite (Eds.), The Internet in everyday life (pp. 215-243). Oxford, England: Blackwell.

Obst, P., y Stafurik, J. (2010). Online we are all able bodied: Online psychological sense of community and social support found through membership of disability-specific websites promotes well-being for people living with a physical disability. Journal of Community \& Applied Social Psychology, 20(6), 525-531. https://doi.org/10.1002/casp.1067

Pavot, W., y Diener, E. (1993). Review of the Satisfaction With Life Scale. Psychological Assessment, 5(2), 164-172.

Peng, Y. (2016). Student migration and polymedia: mainland Chinese students' communication media use in Hong Kong. Journal of Ethnic and Migration Studies, 42(14), 2395-2412. https://doi.org/10.1080/13691 83X.2016.1194743

Quan-Haase, A., Mo, G. Y., y Wellman, B. (2017). Connected seniors: How older adults in East York exchange social support online and offline. Information, Communication \& Society, 20(7), 967-983. https://doi.org/1 0.1080/1369118X.2017.1305428

Quan-Haase, A., e Young, A. L. (2010). Uses and gratifications of social media: A comparison of Facebook and instant messaging. Bulletin of Science, Technology \& Society, 30(5), 350-361. https://doi.org/10.1177/0270467610380009

Ramos-Vidal, I., y Maya-Jariego, I. (2014). Sentido de comunidad, empoderamiento psicológico y participación ciudadana en trabajadores 
de organizaciones culturales. Psychosocial Intervention, 23(3), 169-176. https://doi.org/10.1016/j.psi.2014.04.001

Retortillo, A., y Rodríguez, H. (2010). Estrategias de aculturación y adaptación psicológica en un grupo de inmigrantes. Apuntes De Psicología, 28(1), 19-30.

Salinero-Fort, M. A., Jiménez-García, R., De Burgos-Lunar, C., ChicoMoraleja, R. M., y Gómez-Campelo, P. (2015). Common mental disorders in primary health care: Differences between Latin American-born and Spanish-born residents in Madrid, Spain. Social Psychiatry and Psychiatric Epidemiology, 50(4), 429-443. https://doi.org/10.1007/s00127014-0962-5

Sarason, S. (1974). The Psychological sense of community: Prospects for a community psychology. San Francisco, USA: Jossey Bass.

Şerban, M. (2007). Mecanismos de desarrollo de la migración a nivel de comunidad: Redes de migrantes y tipos de vínculos. Migraciones, 21, $159-188$.

Siegel, S. (1983). Estadística no paramétrica aplicada a las ciencias de la conducta (2a ${ }^{\mathrm{a}} 8^{\mathrm{a}}$ reimp. ed.). México: Trillas.

Suriá, R. (2017). Redes virtuales y apoyo social percibido en usuarios con discapacidad: análisis según la tipología, grado y etapa en la que se adquiere la discapacidad. Escritos de Psicología, 10, 31-40.

Taylor, S. E., Sherman, D. K., Kim, H. S., Jarcho, J., Takagi, K., y Dunagan, M.S. (2004). Culture and social support: Who seeks it and why? Journal of Personality and Social Psychology, 87 (3), 354-362.

Thoits, P. A. (1982). Conceptual, methodological, and theoretical problems in studying social support as a buffer against life stress. Journal of Health and Social Behavior, 23, 145-159.

Trepte, S., Dienlin, T., y Reinecke, L. (2015). Influence of social support received in online and offline contexts on satisfaction with social support and satisfaction with life: A longitudinal study. Media Psychology, 18(1), 74-105. https://doi.org/10.1080/15213269.2013.838904

Valkenburg, P. M., y Peter, J. (2007). Internet communication and its relation to well-being: identifying some underlying mechanisms. Media Psychology, 9(1), 43-58. https://doi.org/10.1080/15213260709336802

Viruela, R. (2007). Migración y nuevas tecnologías de la información y la comunicación: inmigrantes rumanos en España. Migraciones, 21, 259-290.

Walther, J. B., Hoter, E., Ganayem, A., y Shonfeld, M. (2015). Computermediated communication and the reduction of prejudice: A controlled longitudinal field experiment among jews and arabs in Israel. Computers in Human Behavior, 52, 550-558. https://doi.org/10.1016/j. chb.2014.08.004

Wang, E. S., y Wang, M. C. (2013). Social support and social interaction ties on internet addiction: Integrating online and offline contexts. 
Cyberpsychology, Behavior, and Social Networking, 16(11), 843-849. https://doi.org/10.1089/cyber.2012.0557

Wang, J., Hong, J., y Pi, Z. (2015). Cross-cultural adaptation: The impact of online social support and the role of gender. Social Behavior and Personality: An International Journal, 43(1), 111-121. https://doi. org/10.2224/sbp.2015.43.1.111

Wong, W. K.F., Ng, I. F.S., y Chou, K. (2017). Factors contributing to social support among female marriage migrants in Hong Kong: A longitudinal study. International Social Work, 60(2), 394-408. https://doi. org/10.1177\%2F0020872815607955 